\title{
Corrosión de Aleaciones Aeronáuticas de Aluminio y sus Componentes Relacionada a la Expresión Proteica del Hongo Hormoconis resinae
}

\author{
Raquel Araya ${ }^{1}$, Carla V. Bobadilla ${ }^{1}$, Blanca M. Rosales ${ }^{2}$ y Rosa Vera ${ }^{1}$ \\ (1) Pontificia Universidad Católica de Valparaíso, Instituto de Química, Av. Brasil 2950, \\ Valparaíso-Chile. (e-mail: rarayaa@ucv.cl; carlabobadilla@yahoo.com.ar; rvera@ucv.cl) \\ (2) CIDEPINT, Av 52 s/n entre 121 y 122, B1900AYB, La Plata-Argentina \\ (e-mail: brosales@fibertel.com.ar)
}

\begin{abstract}
Resumen
Este artículo muestra que los requerimientos nutricionales de oligoelementos constituyen la fuerza impulsora de la corrosión influenciada microbiológicamente que produce Hormoconis resinae en aleaciones aeronáuticas de aluminio. Se compararon perfiles proteicos de biopelículas del hongo cultivado en turbocombustible aeronáutico mediante electroforesis en geles de poliacrilamida. Se caracterizó la morfología de ataque de las aleaciones y de sus componentes metálicos puros y el crecimiento de hifas sobre cada metal mediante microscopía electrónica de barrido ambiental. Se concluyó que la corrosión estaría asociada a aleantes que actuarían como oligoelementos para el microorganismo.
\end{abstract}

Palabras claves: aleaciones aeronáuticas, Hormoconis resinae, expresión proteica, biopelícula

\section{Corrosion of Aeronautical Aluminium Alloys and its Components Related to the Proteic Expression of the Fungus Hormoconis resinae}

\begin{abstract}
This article shows that the metabolic needs of oligoelements constitute the driving force of the microbiologically influenced corrosion produced by Hormoconis resinae on aeronautical aluminium alloys. Protein profiles of biofilms generated by fungal strains cultivated in aeronautical fuel were compared by electrophoresis in poliacrylamide gels. The morphology attack of alloys, its component metals and the growth of hyphae in cultures were characterized by means of environmental scanning electronic microscopy. One of the main conclusions is that corrosion would be associated to alloying elements that would act as oligoelements for the microorganism.
\end{abstract}

Keywords: aeronautical alloys; Hormoconis resinae, proteic expression, biofilm 


\section{INTRODUCCION}

La corrosión influenciada microbiologicamente (CIM) se refiere a la influencia de los microorganismos sobre la cinética del proceso de corrosión de metales causado por microorganismos que se adhieren a las interfases (usualmente denominados biopelículas). El prerrequisito para CIM es la presencia de microorganismos: si la corrosión es influenciada por su actividad, los otros requerimientos son (I) un electrolito acuoso, (II) una fuente de energía, (III) una fuente de carbono, (IV) un dador de electrones y (V) un aceptor de electrones.

Los microorganismos involucrados en la CIM de metales como hierro, cobre y aluminio y sus aleaciones son fisiológicamente diversos. Bacterias y hongos pueden producir grandes cantidades de ácidos inorgánicos y orgánicos como subproductos metabólicos. Es bien conocido que los hongos producen ácidos orgánicos que son capaces de contribuir a la CIM (Bento et al., 2005).

Muchos de los trabajos publicados en biocorrosión de aluminio y sus aleaciones han sido asociados con la contaminación de combustibles aeronáuticos causados por los hongos Hormoconis (previamente clasificado como Cladosporium) resinae, Aspergillus spp., Penicillium spp. y Fusarium spp. Hormoconis resinae utiliza los hidrocarburos del combustible diesel para producir ácidos orgánicos, siendo los sitios comunes de ataque las superficies en contacto con la fase acuosa de mezclas combustible-agua y sedimentos. La gran cantidad de subproductos orgánicos ácidos excretados por este hongo disuelven o quelan selectivamente cobre, zinc y hierro en los bordes de grano de aleaciones aeronáuticas de aluminio, formando picaduras las cuales persisten en condiciones anaeróbicas formadas bajo el material fúngico (Beech et al., 2000; Sand, 1997).

En la década de los 60 un nuevo problema de corrosión se manifestó en la aviación militar con el reemplazo de la gasolina por kerosene (Churchil, 1963). Este combustible dio lugar al crecimiento de diversas especies microbianas tales como bacterias, levaduras y hongos, dadas las condiciones para su desarrollo: agua y una fuente de carbono adecuada. De esta forma, el hongo filamentoso Hormoconis resinae prolifera causando fallas en el tanque de combustible debido al ataque corrosivo localizado que produce el contacto de su biomasa con las aleaciones aeronáuticas de aluminio (Parbery, 1971; Rosales y Schiapparelli, 1980; Gaylarde et al., 1999; McNamara et al., 2005).

El análisis del perfil proteico de la biopelícula de este hongo y estudios electroquímicos realizados con la fase acuosa de sus cultivos muestran el efecto de Hormoconis resinae sobre las aleaciones aeronáuticas (AA 2024, AA 7005, AA 7075) y sobre aluminio puro. Estos estudios prueban que para la localización del ataque se requiere además del microorganismo y condiciones ambientales favorables, que uno de los componentes de la aleación sea considerado "oligoelemento" para la especie microbiana (Araya et al., 2007).

El efecto de estas aleaciones sobre el micelio de Hormoconis resinae lleva a la expresión de nuevas proteínas que no se aprecian en Hormoconis resinae crecido en ausencia de las aleaciones, lo que se puede atribuir a la presencia de $\mathrm{Zn}, \mathrm{Mg}, \mathrm{Mn}$ y Fe que son componentes de estas aleaciones y que juegan un rol importante en la estructura y función de variadas proteínas y enzimas (Okuyama et al., 1999, Araya et al., 2007).

Hormoconis resinae tiene una gran capacidad de adaptación para sobrevivir y reproducirse, lo que le permite obtener del medio en el que se encuentre los elementos necesarios para su crecimiento, por tanto este microorganismo es capaz de producir metabolitos que captan metales que inducen la formación de diferentes proteínas (Araya et al., 2007). Las evidencias experimentales demuestran que el intenso ataque de las aleaciones de aluminio en las zonas de contacto con las hifas de Hormoconis resinae es consecuencia de su actividad metabólica ya que su desplazamiento poligonal aumenta la velocidad de corrosión en áreas asociadas a segundas fases de las aleaciones.

La formación de segundas fases $\left(\mathrm{Al}_{2} \mathrm{Cu}\right)$ produce un empobrecimiento de $\mathrm{Cu}$ y $\mathrm{Al}$ y a su vez un enriquecimiento de Fe y $\mathrm{Zn}$ que son por tanto los metales que se disuelven (Rosales, 2000). El Cr es tóxico para el microorganismo, lo mismo que el $\mathrm{Cu}$ y $\mathrm{Ni}$ en cuya presencia no esporula (Rosales y Esteso, 1990). 
La importancia de comprobar si la expresión de estas proteínas por el hongo Hormoconis resinae es inducida por la presencia de uno de los oligoelementos presentes en las aleaciones de base Aluminio o a un conjunto de ellos actuando sinérgicamente, sería útil para controlar el ataque localizado producto de biopelículas formadas por microorganismos que crecen en contacto con aleaciones de base Aluminio que conforman estructuras aeronáuticas; su gravedad reside en que se trata de un ataque muy localizado en la zona donde se adhieren las colonias y que penetra formando fisuras que pueden dar lugar a su perforación o propagarse ocasionando roturas del tipo de las encontradas en casos de fatiga de aleaciones (Rosales y Esteso, 2000).

El propósito de este trabajo fue determinar qué elementos componentes de aleaciones aeronáuticas de base aluminio son responsables de la expresión de proteínas por Hormoconis resinae en la presencia en el medio de cultivo de aleaciones, AA7005, AA7075 y AA2024.

\section{PROCEDIMIENTO EXPERIMENTAL}

Cultivo del hongo Hormoconis resinae en ausencia y presencia de aleaciones aeronáuticas de aluminio.

El hongo se hizo crecer en medio de cultivo Bushnell-Haas modificado (Bushnell y Haas, 1941) diluido 1:10 en equilibrio con combustible aeronáutico Jet $A 1$ (kerosene) como única fuente de carbono para el crecimiento microbiano, en presencia y ausencia de aleaciones aeronáuticas de aluminio, AA 2024, AA 7005 y AA 7075, durante 30 días a $30^{\circ} \pm 1^{\circ} \mathrm{C}$.

Cultivo del hongo Hormoconis resinae en presencia de iones y metales puros componentes de la aleación.

El hongo se hizo crecer por separado en presencia de metales puros y concentraciones de iones oligometálicos equivalentes a las que se encuentran presentes en la aleación de aluminio AA 7005 estudiada ( $\mathrm{Zn}, \mathrm{Cu}, \mathrm{Mn}, \mathrm{Si}, \mathrm{Mg}, \mathrm{Fe}, \mathrm{Cr}$ ), ajustándose el pH inicial a 5.4, durante 30 días a $30^{\circ} \pm 1^{\circ} \mathrm{C}$. Las sales utilizadas para este propósito fueron: $\mathrm{CuSO}_{4} \times 5 \mathrm{H}_{2} \mathrm{O}, \mathrm{MnSO}_{4} \times \mathrm{H}_{2} \mathrm{O}, \mathrm{FeSO}_{4} \times 7 \mathrm{H}_{2} \mathrm{O}$, $\mathrm{Cr}\left(\mathrm{NO}_{3}\right)_{3} \times 9 \mathrm{H}_{2} \mathrm{O}, \mathrm{MgSO}_{4} \times \mathrm{H}_{2} \mathrm{O}, \mathrm{ZnSO}_{4} \times 7 \mathrm{H}_{2} \mathrm{O}$

\section{Obtención del homogenado}

La biomasa obtenida a partir de los cultivos de Hormoconis resinae fue secada, molida, pesada y luego digerida con diferentes soluciones: detergentes (Twin 20 0.5\%, SDS 3.5\%, DTT $60 \mathrm{mM}$ ), complejantes (EDTA $1 \mathrm{mM}, \mathrm{MgCl}_{2} 1.5 \mathrm{mM}$ ), preservantes (Glicerol 3.0\%) e inhibidor de proteasas (PMSF 5.0mM) durante 24 horas a $4^{\circ} \mathrm{C}$.

\section{Cuantificación de proteínas}

Se utilizó el método de MicroBradford (Bradford, 1976) para la determinación de proteínas presentes en las muestras de homogenados provenientes de los cultivos de Hormoconis resinae y se usó como estándar Albúmina de Suero de Bovino (BSA) de concentración $0.095 \mathrm{mg} / \mathrm{mL}$. La absorbancia de las muestras se leyó en un espectrofotómetro Shimadzu UV-VISIBLE,UV-1601, a una longitud de onda de $595 \mathrm{~nm}$.

\section{Caracterización de proteínas presentes en el homogenado}

Se realizó electroforesis en gel de poliacrilamida-SDS con marcadores de peso molecular estandarizados para analizar el patrón de proteínas de las diferentes muestras de homogenados con tinción plata para visualizar el perfil de bandas proteicas (Laemmli, 1970).

\section{Curvas de polarización}

En la determinación del comportamiento electroquímico de las aleaciones se utilizaron como electrolitos las fases acuosas de los cultivos del hongo, en presencia y ausencia de las aleaciones 
aeronaúticas de aluminio. Como blanco de las curvas de polarización de las aleaciones, anódicas deaireadas y catódicas saturadas en aire, se usó medio de cultivo Bushnell-Haas estéril, diluído a 1:10 con agua destilada para proveer el medio pobre en nutrientes de los trabajos previos. El intervalo de potenciales fue entre $-2000 \mathrm{mV} \mathrm{y}+2000 \mathrm{mV}$ a una velocidad de barrido de $100 \mathrm{mV} \mathrm{min}^{-1}$. A partir de las curvas de polarización se determinaron los potenciales de picado $\left(E_{p}\right)$ de las aleaciones (Schiapparelli y Rosales, 1980; Rosales et al., 1993; Bento et al., 2004).

\section{Ensayo a la gota}

Para visualizar el ataque superficial se realizaron ensayos a la gota con el micelio de los diferentes cultivos en cada una de las aleaciones (Hansen et al., 1981; Zinkevich et al., 1996). Se analizó la mutua interacción por el contacto durante el crecimiento directo del hongo sobre la aleación. La terminación superficial de estas muestras se obtuvo puliendo con papel esmeril grado 600 .

\section{Microscopía electrónica de barrido ambiental y EDAX}

Se efectuaron análisis de microscopía electrónica de barrido ambiental y de Energía dispersiva de Rayos-X, MEBA-EDAX, del aspecto metal-biopelícula para:

a) Caracterizar la susceptibilidad de las aleaciones aeronáuticas a la CIM sometidas al ensayo a la gota luego de 7 a 10 días de contacto con el micelio.

b) Observar la morfología metal-biopelícula en los metales puros constituyentes de las aleaciones aeronáuticas de aluminio utilizados en cultivos de Hormoconis resinae.

\section{RESULTADOS Y DISCUSIÓN}

El análisis de las proteínas de los homogenados de la biomasa de Hormoconis resinae revela diferencia en los perfiles proteicos para el micelio crecido en presencia o ausencia de las aleaciones. (Figura 1).

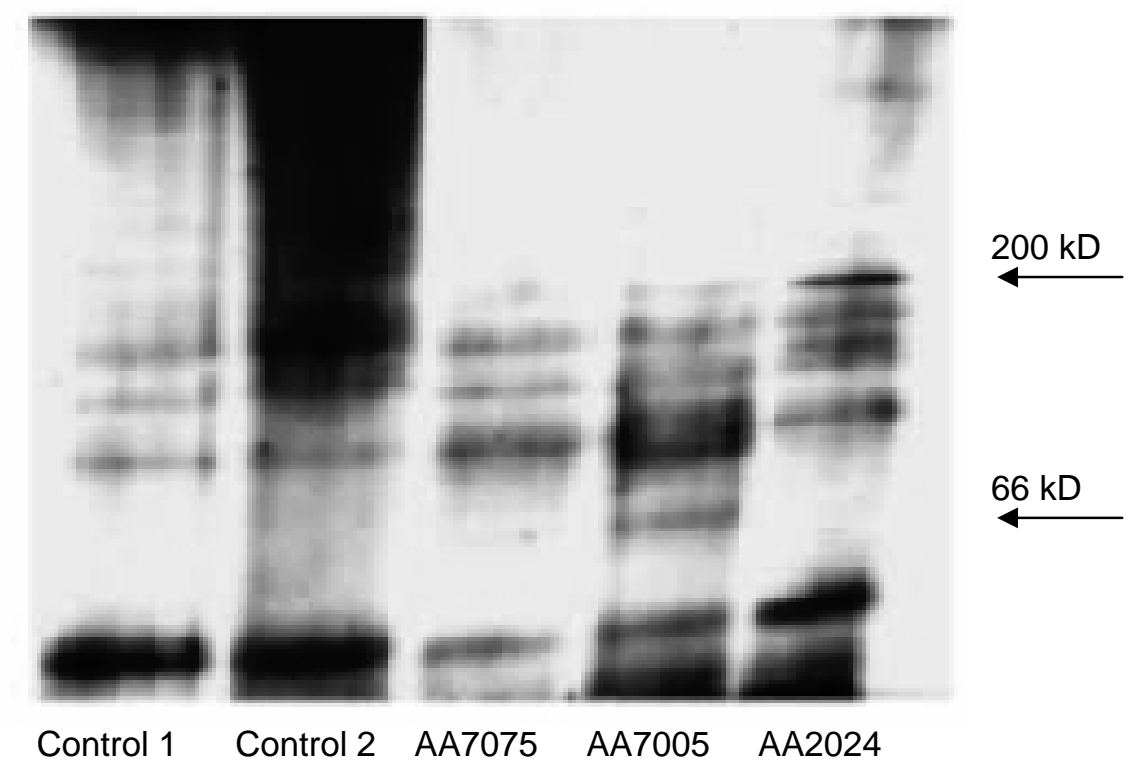

Fig. 1: Electroforesis de proteínas del micelio Hormoconis resinae, crecido en presencia y ausencia de las aleaciones.

El hongo en presencia de las aleaciones expresa una banda proteica con peso molecular a $200 \mathrm{KD}$ que es más evidente en AA2024, sin embargo la mayor diferencia se aprecia en el micelio formado en presencia de AA7005 debido a que aparece una banda de peso molecular de alrededor de 66 KD que no es expresada por el hongo en presencia de las otras aleaciones. 
La polarización de las aleaciones de aluminio realizadas en los medios de cultivo (al cabo de 30 días de incubación), inoculados en ausencia y presencia de las aleaciones revelan la intensificación del efecto de la CIM del hongo con una disminución del potencial de picado ( $\left.E_{p}\right)$ desde aproximadamente 2000 mVcs a 1500 mVcs para AA 7075 y desde 2000 mVcs a 1000 mVcs para AA 2024 (Figura 2).

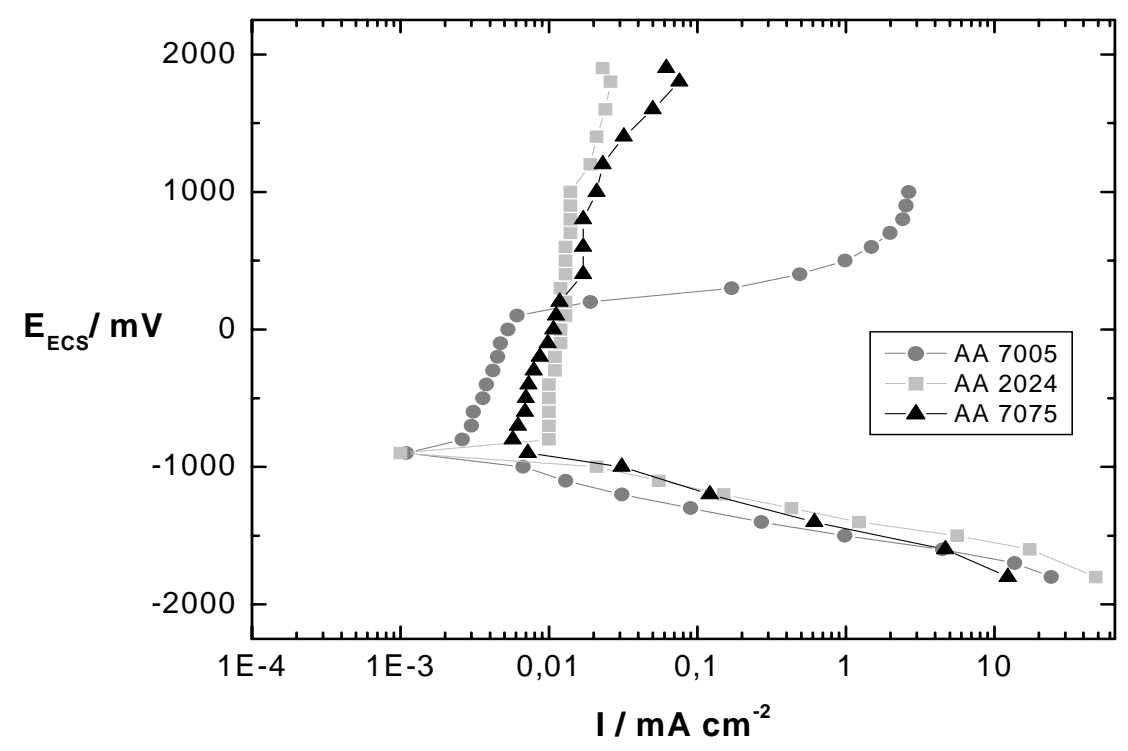

a)

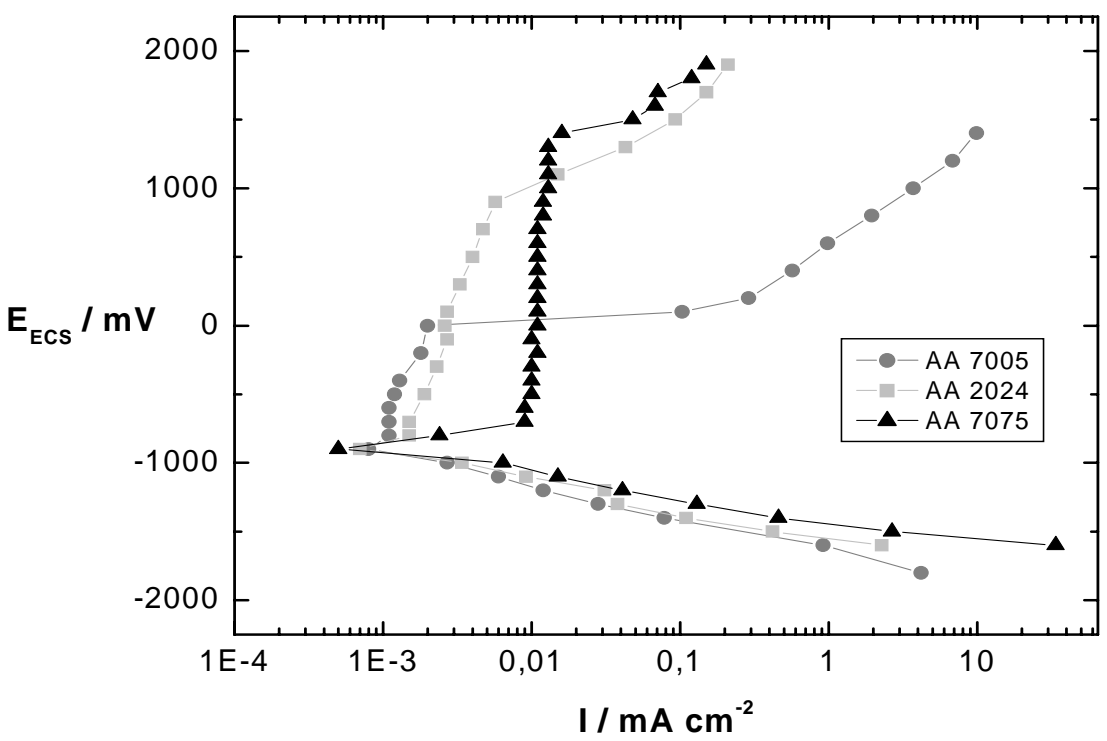

b)

Fig. 2: Curvas de Polarización de las aleaciones aeronáuticas de aluminio en la fase acuosa de los cultivos de Hormoconis resinae en a) ausencia y b) presencia de la respectiva aleación.

Los resultados electroquímicos demuestran que el crecimiento del micelio en presencia de estas aleaciones incrementa su susceptibilidad a la rotura de la pasividad, la que se manifiesta en su disminución del respectivo potencial de picado. La presencia de AA7005 en el cultivo, por el contrario, no afecta prácticamente la susceptibilidad a la MIC por la cepa.

Ensayos a la gota realizados luego de cinco meses de almacenamiento del hongo, muestran que el micelio de hongo aplicado produce ataque en la superficie de las aleaciones. En la Figura 3 se pueden observar las diferentes morfologías encontradas mediante microscopía electrónica de barrido ambiental (MEBA): Al puro no fue afectado y se nuclearon ataques cristalográficos en todas las aleaciones de aluminio. En AA2024 los ataques fueron preferenciales sobre segundas fases y el 
análisis EDAX demuestra en la Tabla 1, cuales fueron los más susceptibles. Evidencias similares han sido expuestas por otros autores (Ayllón et al., 1994).

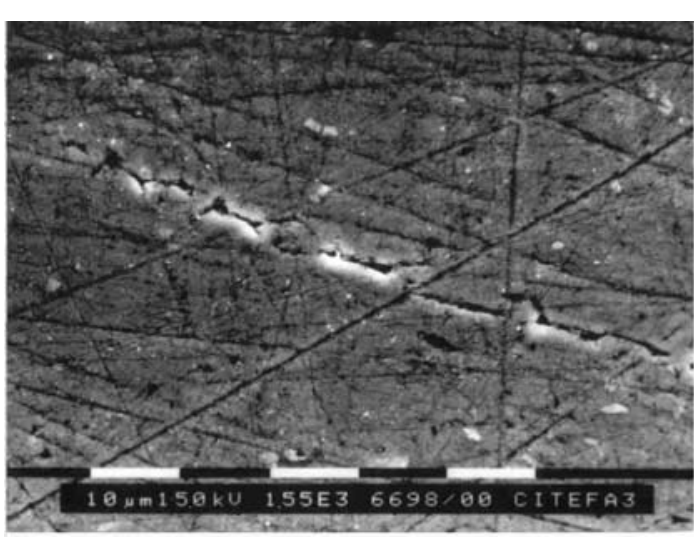

a)

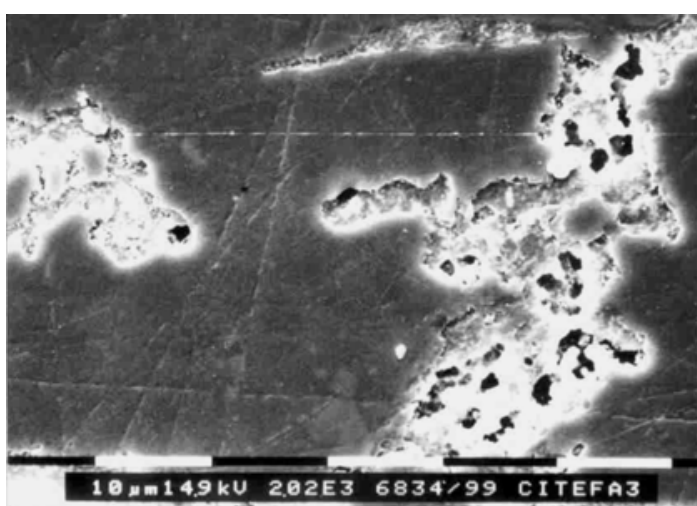

c)

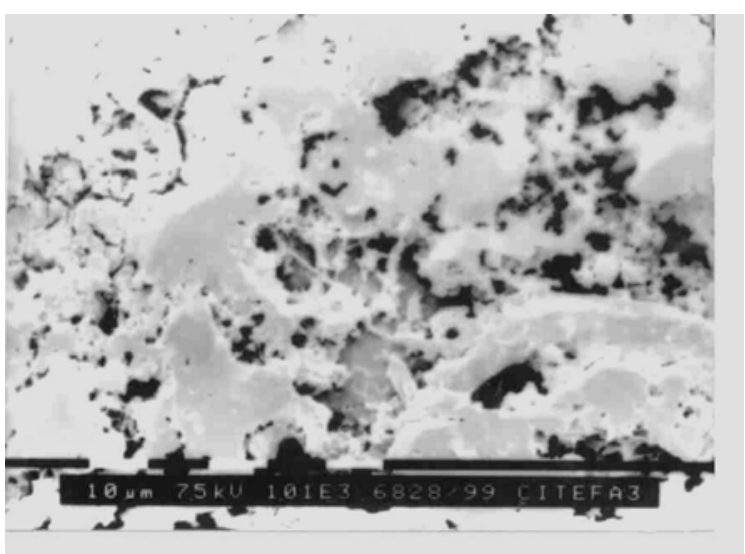

b)

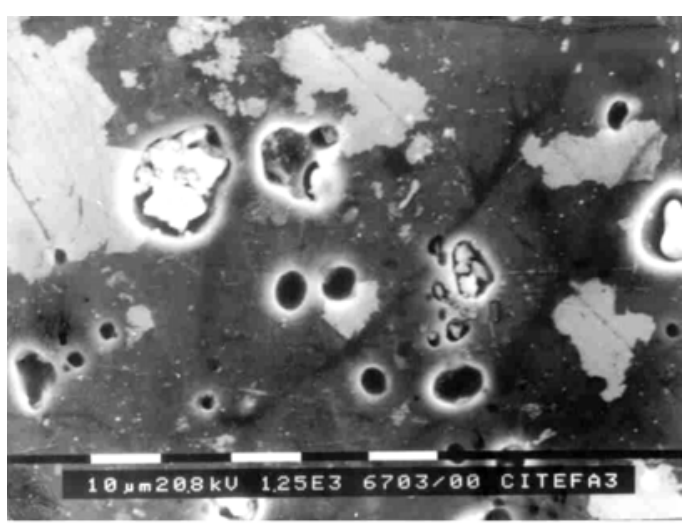

d)

Fig. 3: Aspecto MEBA de muestras metálicas sometidas al ensayo de gota con micelio de Hormoconis resinae: a) Al 99.99\% (1500x); b) AA7075 (1000x); c) AA7005 (2000x) y

d) AA2024 (1250x)

Tabla 1: EDAX de segundas fases sobre la matriz de la aleación AA2024 x 5000

\begin{tabular}{|c|c|c|c|c|c|c|}
\hline $\begin{array}{c}\text { Elementos \% } \\
\text { en Peso }\end{array}$ & \multicolumn{4}{|c|}{ Forma redonda } & $\begin{array}{c}\text { Forma } \\
\text { Irregular }\end{array}$ & Matriz \\
\hline $\mathrm{Al}$ & 74.00 & 80.24 & 60.63 & 56.15 & 37.76 & 98.88 \\
\hline $\mathrm{K}$ & 2.15 & - & - & - & 4.35 & - \\
\hline $\mathrm{Cu}$ & 24.00 & 15.92 & 39.37 & 4.09 & 40.35 & 1.12 \\
\hline $\mathrm{Fe}$ & - & 3.85 & - & - & 13.79 & - \\
\hline $\mathrm{Si}$ & - & - & - & 39.76 & - & - \\
\hline $\mathrm{S}$ & - & - & - & - & 3.75 & - \\
\hline
\end{tabular}

La biomasa de Hormoconis resinae obtenida de los cultivos realizados en presencia de $\mathrm{Mg}^{2+}, \mathrm{Cr}^{3+}$, $\mathrm{Fe}^{2+}, \mathrm{Zn}^{2+}$ y $\mathrm{Cu}^{2+}$ se mantuvo en el mismo peso que el inóculo de micelio sembrado inicialmente. Ello sugiere que los iones adicionados al medio no estimularon el crecimiento del hongo o bien que Hormoconis resinae no reveló necesidad metabólica alguna que diera lugar a su captación como nutrientes. En contraste, en presencia de Fe, Mg y $\mathrm{Zn}$ en sus formas metálicas se obtuvo mayor biomasa del hongo y los metales evidenciaron abundantes productos de corrosión. La morfología de la superficie de los metales fue observada mediante Microscopía Electrónica de barrido ambiental, Figura 4, con diversas magnificaciones según sus variadas morfologías y tamaños, los cuales no se intentó comparar. 


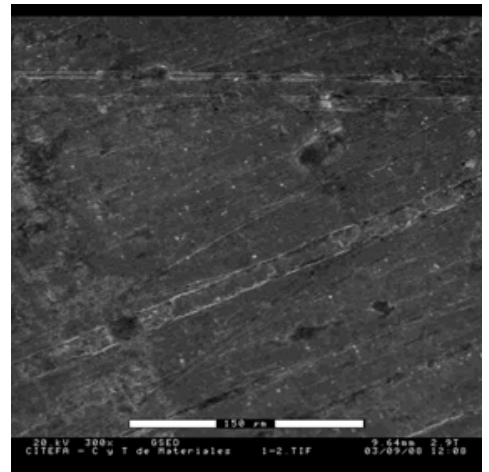

a)

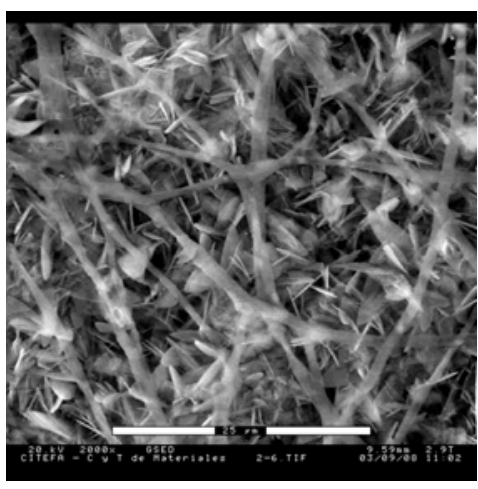

d)

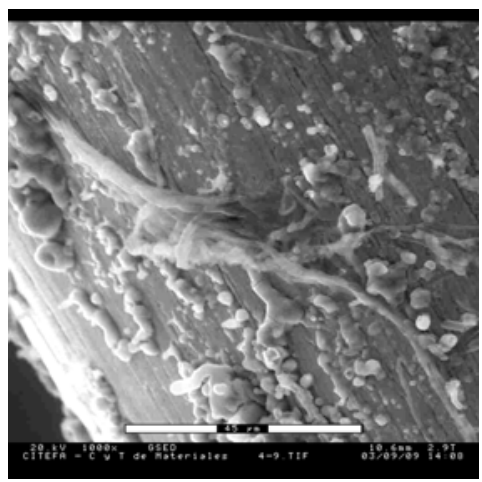

g)

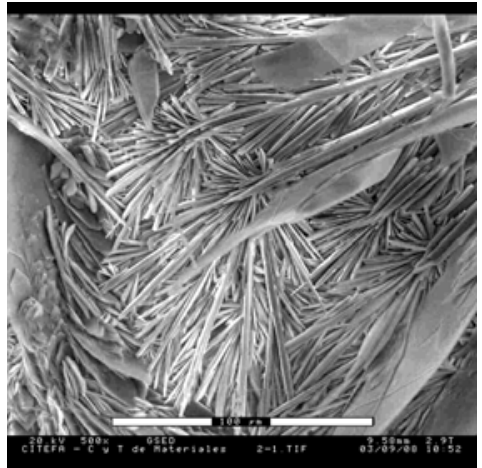

b)



e)

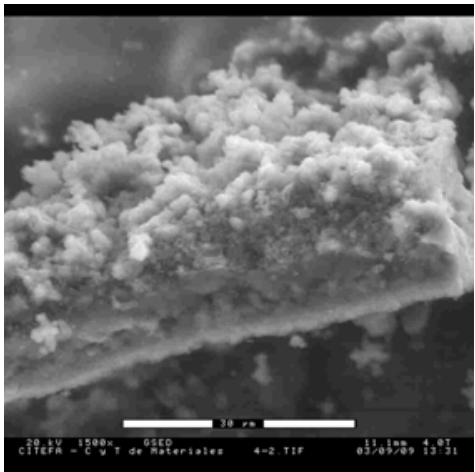

h)



c)

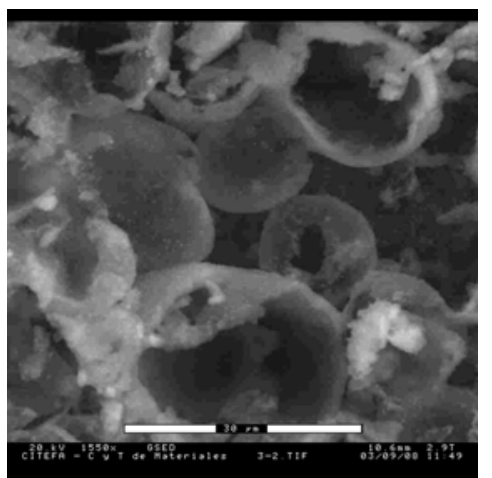

f)

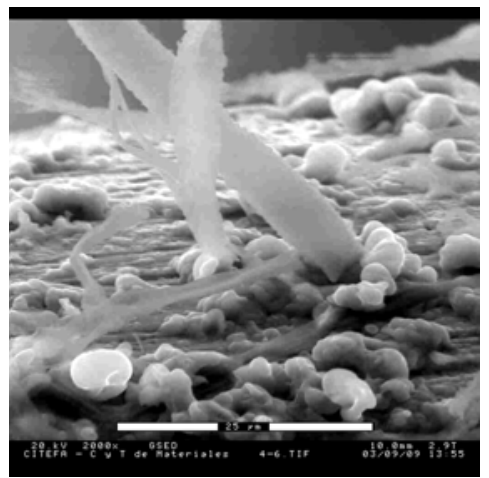

i)

Fig. 4: Aspecto MEBA de los productos de corrosión de los metales constituyentes de las aleaciones aeronáuticas de aluminio en cultivos de Hormoconis resinae: a) Cu (300x); b) Zn (500x) c) Zn (550x); d) Zn (2000x); e) Mg (600x); f) Mg (1550x); g) Fe (1000x); h) Fe (1500x); i) Fe (2000x)

Se observa que la hifa del hongo no atacó ni quedó adherida a la muestra de cobre. Sólo se ve su huella, a diferencia de la fuerte interacción que se puede apreciar sobre $\mathrm{Fe}, \mathrm{Zn}$ y $\mathrm{Mg}$, en donde el micelio del hongo, a pesar de haber sido retirado se fijó de tal forma al metal, que siguió creciendo, nutriéndose de la red metálica a su disposición y generando abundantes productos de corrosión. El cobre no es susceptible de ser corroído, a diferencia de Fe, Mg y Zn.

En la Figura 5, se muestra el perfil proteico obtenido de los cultivos de Hormoconis resinae en presencia de los metales componentes de AA7005.

De los resultados del análisis de los perfiles proteicos obtenidos de los homogenados de Hormoconis resinae cultivados en presencia de los metales puros componentes de las aleaciones, se deduce que la expresión de nuevas proteínas debida a alguno de ellos, tales como Fe y Zn, podrían ser las 
responsables de la corrosión observada al retirar las chapas metálicas de sus respectivos cultivos. Sólo en presencia de $\mathrm{Cu}$, Hormoconis resinae no presenta ninguna de las bandas de proteínas que caracterizan al control. Los perfiles obtenidos para $\mathrm{Cu}$ y $\mathrm{Zn}$ presentan similitud, sólo una banda de proteína diferente $(34.3 \mathrm{KDa})$ presente en Cu podría ser la responsable de algún mecanismo de autodefensa del microorganismo ante la toxicidad que le causa el cobre, lo cuál se evidencia en la inhibición del crecimiento del hongo en presencia de este metal. Los perfiles para los homogenados obtenidos de cultivos en presencia de $\mathrm{Mg}$, Fe y $\mathrm{Zn}$, arrojan resultados de 4, 3 y 2 bandas coincidentes con el perfil del control respectivamente, lo que se atribuye a que estos metales son oligoelementos necesarios para el metabolismo celular del microorganismo. Por lo tanto la corrosión observada en aleaciones de base aluminio como la AA7005 (ver figura 3c) podría deberse a que el hongo, al crecer en presencia de esta aleación, revela su capacidad de captar estos elementos metálicos de la red cristalina de la aleación provocando las diversas formas de corrosión observadas, con predominio del picado cristalográfico (Araya et al., 2002).

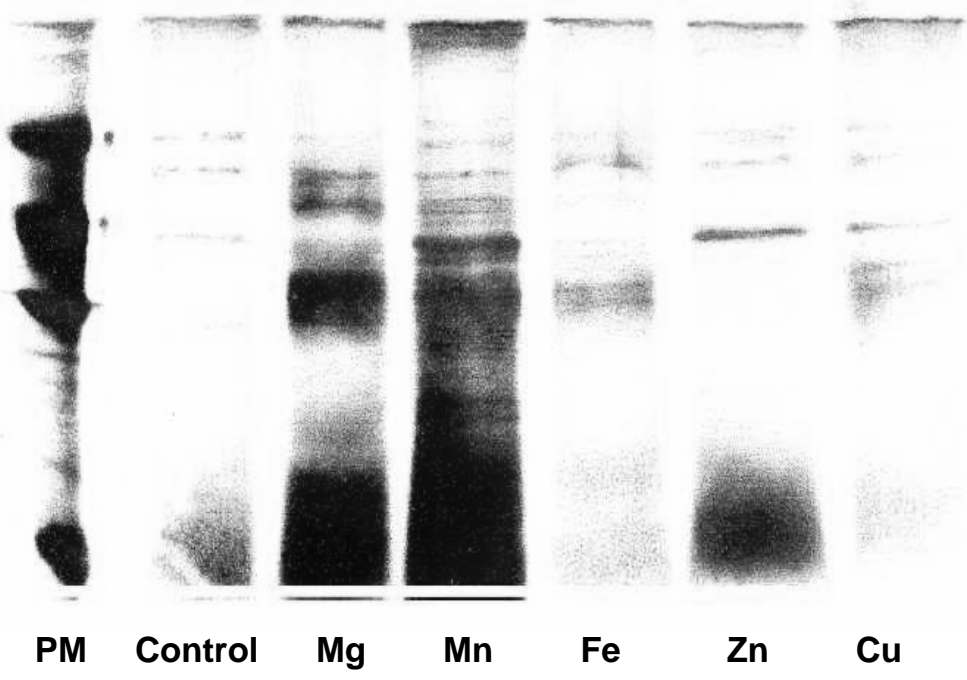

Fig. 5: Electroforesis de proteínas de Hormoconis resinae en presencia de los aleantes de AA7005.

\section{CONCLUSIONES}

Los efectos que produce Hormoconis resinae en la corrosión de aleaciones aeronáuticas de aluminio pueden atribuirse a la expresión de proteínas que son inducidas cuando el hongo crece en presencia de las aleaciones. La proteína representada en la banda electroforética de 200 kD para AA2024, AA7005 y AA7075, sería responsable de la captación de oligoelementos de la red metálica, a la vez que estimula el crecimiento celular.

La proteína de 66 kD expresada en presencia de la aleación AA7005 sugiere que el microorganismo es inducido por los componentes de esta aleación a producir una proteína que lo hace ser menos agresivo frente a la corrosión, lo que explicaría que la susceptibilidad a la CIM de AA7005 no se ve afectada al utilizar medios de cultivos en que Hormoconis resinae creció en presencia de esta aleación.

De los perfiles proteicos, así como de los ensayos electroquímicos y a la gota se revela una interacción específica del hongo con cada superficie metálica, lo que justificaría la agresividad de Hormoconis resinae sobre las aleaciones aeronáuticas estudiadas.

Hormoconis resinae manifiesta mayor afinidad hacia los oligoelementos presentes en una red cristalina metálica que cuando se encuentran en forma de sales solubles. Esta afinidad por obtener bioelementos desde la red metálica estaría asociada al consumo de electrones, que utilizaría en su metabolismo energético (síntesis de ATP). 
En las aleaciones aeronáuticas analizadas, el hongo capta selectivamente iones de la red metálica provocando picaduras en las zonas en las que estos elementos están presentes.

\section{AGRADECIMIENTOS}

A la Dirección de Investigación e Innovación de la Pontificia Universidad Católica de Valparaíso por el financiamiento del proyecto DII 125.758 .03

\section{REFERENCIAS}

Araya, R., B.M. Rosales, C.V. Bobadilla y R. Vera; MIC of aeronautical aluminium alloys by a strain of Hormoconis resinae, Actas $15^{\circ}$ Congreso Internacional de Corrosión, Paper №780, Granada, España, (2002).

Araya, R., C.V. Bobadilla, R. Vera y B.M. Rosales; Biochemical analysis of the Hormoconis resinae fungal mycelium in the corrosion of aeronautical aluminium alloys, Revista de Metalurgia: 43(3), 228236 (2007).

Ayllón, E.S. y B.M. Rosales; Electrochemical test for predicting microbiologically influenced corrosion of aluminum and AA7005 alloy, Corrosion Science: 50 (8), 571-575 (1994).

Beech, I. y otros cinco autores; "Simple Methods for the Investigation of the role of Biofilms in Corrosion" en Brite Euram Thematic Network on MIC of Industrial Materials No ERB BRRT-CT985084, Biocorrosion 00-02, Septiembre (2000).

Bento, F.M. y otros cuatro autores; "Degradation and corrosive activities of fungi in a diesel-mild steelaqueous system", World Journal of Microbiology \& Biotechnology: 21, 135-142 (2005).

Bento, F.M., G.E. Englert, C.C. Gaylarde e I.L. Muller; Influence of aqueous phase on electrochemical biocorrosion tests in diesel/water systems, Materials and Corrosion: 55 (8), 577-585 (2004).

Bradford, M.M.; A rapid and sensitive method for the quantitation of microgram quantities of protein utilizing the principle of protein-dye binding, Anal Biochem.: 72, 248-254 (1976).

Bushnell, L.D. y H.F. Haas; The utilization of certain hidrocarbons by microorganisms, J. Bacteriology: 41, 653-673 (1941).

Churchil, A.V.; Microbial fuel tankcorrosion mechanisms and contributing, Materials Protection: 2(1), 19-28 (1963).

Gaylarde, C.C., F.M. Bento y J. Kelley; Microbial Contamination of Stored hydrocarbon fuels and its control, Revista de Microbiología: 30, 01 -10 (1999).

Hansen, D.J., D.J. Tighe-Ford y G.C. George; Role of the mycelium in the corrosive activity of Cladosporium resinae in a Dieso-water system. International Biodeterioretion Bulletin: 17(4), 103-112 (1981).

Laemmli, U.K.; Cleavage of Structural Proteins during the assembly of the Head of Bacteriophage T4, Nature: 227, 680- 685 (1970).

McNamara, C.J. y otros cinco autores; Corrosion of aluminum alloy 2024 by microorganisms isolated from aircraft fuel tanks, Biofouling: 21(5/6), 257-265 (2005).

Okuyama, M. y otros cuatro autores; Effect of some heavy metal ions on copper-induced metallothionein synthesis in the yeast Saccharomyces cerevisiae, Biometals: 12(4), 307-314 (1999). 
Parbery, D.G.; Biological problems in jet aviation fuel and the biology of Amorphoteca resinae, Materials und Organismen: 6(3), 161-208 (1971).

Rosales, B.M. y E.R. de Schiapparelli; A corrosion test for determining the quality of maintenance in jet fuel storage, Materials Performance: 19(8), 41-44 (1980).

Rosales, B.M., A. Puebla y D. Cabral; Role of the metal uptake by the mycelium of the fungus Hormoconis resinae in the MIC of Al alloys, Actas XI Congreso Internacional de Corrosión, Houston, USA, 5B, 3773 (1993).

Rosales, B.M. y M.A. Esteso; Microbial corrosion of pure metals under fungal biofilms, Actas $11^{\circ}$ Congreso Internacional de Corrosión, 359(4), Florencia, Italia (1990).

Rosales, B.M.; "Influencia de la biopelícula del hongo Hormoconis resinae en la corrosión microbiológica de aleaciones de aluminio" en Curso de Corrosión Microbiológica en la Industria, Pontificia Universidad Católica de Valparaíso, Noviembre (2000).

Sand, W.; Microbial mechanisms of deterioration of inorganic substrates - A general mechanistic overview, International Biodeterioration \& Biodegradation: 40 (2-4), 183-190 (1997).

Schiapparelli, E.R. y B.M. Rosales; Microbiological corrosion in terminal storage tanks for aircraft fuel. Materials Performance: 19(10), 47-50 (1980).

Zinkevich, V. y otros cinco autores; Characterisation of Exopolymers Produced by Different Isolates of Marine Sulphate-reducing Bacteria, International Biodeterioration \& Biodegradation: 163-172 (1996). 